definierten Gleichungssystems scheinen dem Verf. ein starkes Argument dafür, daß die wirkliche Theorie der Elementarteilchen durch einen solchen Formalismus dargestellt wird. Die große Mannigfaltigkeit der Elementarteilchen würde damit auf ein einfaches Gleichungssystem zurückgeführt, in dem als Dimensionskonstante nur noch die „kleinste Länge“ $l$ vorkommt. Alle Elementarteilchen wären nur stationäre Zustände ein und derselben „Materie“.

Unbefriedigend bleibt einstweilen noch die große Willkür, die bei der Wahl der Funktionen $S_{\alpha \beta}^{R}$ und $H(x)$ zu bestehen scheint; aber man kann hoffen, daß die richtigen Funktionen durch besondere Eigenschaften, etwa durch Einfachheit oder durch Invarianz bei bestimmten Transformationen, ausgezeichnet sind. Die Aufgabe der nächsten Zeit wird eben darin bestehen, aus den empirischen Eigenschaften der Elementarteilchen Schlüsse auf die Strukturen der Funktionen $S_{\alpha \beta}^{R}$ und $H$ zu ziehen. Zum Verständnis dieser Strukturen gehört z. B. auch das Verständnis der Eichtransformation und des mit ihr offenbar verbundenen Wertes von $e^{2} / \hbar c$; denn die ganze Quantenelektrodynamik sollte ja nur ein Ausschnitt aus dem Gleichungssystem (1) und (8) sein.

\title{
Über die Konzentrationsabhängigkeit von Volumeneffekt und Dipolmoment binärer und ternärer Gemische einiger organischer Flüssigkeiten
}

\author{
Von Maria Joerges, geb. Heyden und Alexander Nikuradse \\ Aus dem Institut für Elektronen- und Ionenforschung an der Technischen Hochschule München \\ (Z. Naturforschg. 5 a, 259-269 [1950]; eingegangen am 9. Februar 1950)
}

An binären und ternären Gemischen von Hexan, Methylalkohol und Chlorbenzol sowie von Schwefelkohlenstoff, Methylalkohol und Aceton werden Volumeneffekt und Dielektrizitätskonstante bei $20^{\circ} \mathrm{C}$ bestimmt. Aus den DK-Messungen werden nach der Debye-ClausiusMosottischen und nach der Onsagerschen Formel die Dipolmomente berechnet. Aus ihrer Konzentrationsabhängigkeit und dem Verlauf des Volumeneffektes wird auf den Assoziationszustand geschlossen.

Speziell läßt sich für Aceton-Methylalkoholgemische aus dem nach Onsager berechneten Dipolmoment eine $\mathrm{OH} \ldots \mathrm{O}=\mathrm{C}$-Brückenbildung ablesen.

$\mathrm{D}$ er Volumeneffekt besteht in einer VolumenKontraktion oder -Dilatation, die meist beim Mischen von Flüssigkeiten eintritt. Die Dielektrizitätskonstante einer Substanz ist eine Funktion der Polarisierbarkeit, deren Größe und Verhalten vor allem dann von Interesse ist, wenn es sich um Moleküle handelt, die ein permanentes elektrisches Moment tragen. Untersucht man nun an den gleichen Gemischen organischer Flüssigkeiten Dielektrizitätskonstante und Volumeneffekt, so steht zur Diskussion, ob und in welcher Weise diese beiden Funktionen molekularer Eigenschaften miteinander verknüpft sind. Schon früher sind darüber von K. L. Wolf und Mitarbb. Úberlegungen angestellt worden. Sie stützten sich aber hinsichtlich des Verhaltens der polaren Substanzen lediglich auf die Debyesche Theorie und die Debye-Clausius-Mosottische Formel zur Berechnung von Dipolmomenten gasförmiger Substanzen. Es konnte sich daher keine allgemein befriedigende Deutung ergeben. Die von Onsager und später von $\mathrm{K}$ irkw o od speziell für das flüssige Dielektrikum entwickelte Theorie, die früher wenig beachtet und erst in jüngster Zeit durch die Arbeiten von M e cke und seinen Schülern aufgegriffen und eingehender Kritik unterworfen wurde, hat ergeben, daß sie gerade dann von besonderer Bedeutung ist, wenn es sich nicht um die immer zwischen Dipolen auftretenden Attraktionskräfte handelt, sondern um eine spezifische, also strukturabhängige zwischenmolekulare Wechselwirkung gleicher oder ungleicher Moleküle untereinander.

$\mathrm{Zu}$ den im folgenden berichteten Untersuchunge: wurden binäre und ternäre Gemische aus Schwefelkohlenstoff, Aceton und Methylalkohol und außerdem Gemische aus Hexan, Chlorbenzol und Methylalkohol herangezogen. Von ihnen sind bekanntlich Aceton, Chlorbenzol und Methylalkohol Dipolsubstanzen, während Schwefelkohlenstoff und Hexan unpolare Flüssigkeiten sind. 


\section{a) Der Volumen-Effekt}

Stellt man ein Gemisch aus $x_{\mathrm{A}}$ Molanteilen der Substanz A und aus $x_{\mathrm{B}}$ Molanteilen der Substanz B her, wo $x_{\mathrm{A}}+x_{\mathrm{B}}=1$ sein soll, so sollte das Volumen

$$
x_{\mathrm{A}} M_{\mathrm{A}} / \varrho_{\mathrm{A}}+x_{\mathrm{B}} M_{\mathrm{B}} / \varrho_{\mathrm{B}}=x_{\mathrm{A}} V_{\mathrm{A}}+x_{\mathrm{B}} V_{\mathrm{B}}
$$

betragen ${ }^{2}$. Tatsächlich findet aber i. allg. eine Volumendilatation $(\Delta V>0)$ bzw. eine Volumenkontraktion $(\Delta V<0)$ statt. Außer den hier angegebenen Messungen sind schon früher von anderen Autoren ${ }^{1}$ Volumeneffekte bestimmt worden. Dabei ließ sich eine Systematik in dem Verlauf der Volumeneffekte binärer Gemische erkennen, die aber keineswegs auf ternäre Gemische erweitert werden konnte. Offenbar liegen auf diesem Gebiet noch keine systematischen Untersuchungen vor.

Die Volumeneffekte von Gemischen aus unpolaren Substanzen sind entweder unmeßbar klein oder aber positiv, jedenfalls dann, wenn sich die Größen der Moleküle nicht zu sehr voneinander unterscheiden. Diese Volumendilatationen dürften in erster Linie auf einem Packungseffekt, also auf eine geometrische Ursache zurückzuführen sein. Zwischenmolekulare Kräfte sind bei unpolaren Flüssigkeiten sicher auch vorhanden, doch sind sie von geringerer Größenordnung als bei polaren Substanzen; wir wollen sie daher nicht berücksichtigen. Dies ist auch insofern berechtigt, als derartige Gemische nur sehr geringe oder verschwindend kleine Mischungswärmen zeigen.

Die $\Delta V$ werden i. allg. mit Hilfe von Dichtemessungen bestimmt, und die Größenordnung des molaren Volumeneffektes beträgt $\sim 0,5 \mathrm{~cm}^{3}$ am Maximum $\left(x_{\mathrm{A}}=x_{\mathrm{B}}\right)$ für Gemische unpolarer Substanzen.

Sehr verschiedenartige Volumeneffekte hinsichtlich des Vorzeichens, der Größe und der Lage des Maximums findet man dann, wenn polare Substanzen als Systemkomponenten vorhanden sind. Meist läßt der Verlauf der Volumeneffekte Rückschlüsse auf das Verhalten der Moleküle zu. Doch liegt eine Schwierigkeit darin, daß der Volumeneffekt hier sicher durch einen geometrischen Packungseffekt und durch zwischenmolekulare Kraftwirkungen zustande kommt. Wird in Gemischen polar-unpolar ein großer positiver Effekt gemessen (z. B. $1 \mathrm{~cm}^{3}$ beim Maximum), so kann man sicher sein, daß die Volumenvergrößerung außer durch den Packungseffekt durch eine Ent-

1 H. H a rms, Diss. Würzburg 1937. K. L. W olf, H. Frahm u. H. Harms, Z. physik. Chem. (B) [1937] 237.

2 Durchgehend ist unter der Bezeichnung $V$ das Molvolumen $\mathrm{zu}$ verstehen. assoziation der polaren Flüssigkeit hervorgerufen wird (z. B. Aceton-Schwefelkohlenstoff mit $\Delta V \max$ $\left.\sim 1 \mathrm{~cm}^{3}\right)$. Ist dagegen der Volumeneffekt negativ, so läßt sich schließen, daß dies die Folge einer neuauftretenden zwischenmolekularen Attraktion ist. Für Gemische polar-unpolar würde das die Bildung von Solvatationskomplexen bedeuten, die auf der Induktionswirkung der polaren Moleküle auf die unpolaren beruht. Gleichzeitig kann man annehmen, daß die Dipolsubstanz selbst nur wenig assoziiert ist; andernfalls müßte der Solvatation eine volumenvergrößernde Entassoziation der polaren Substanz vorangegangen sein. Die Volumenkontraktion würde dann aber aussagen, daß sowohl der Packungseffekt als auch die Volumenänderung durch Entassoziation durch die der Solvatation überkompensiert wird. Das aber ist recht unwahrscheinlich, und man muß dabei bleiben, derartige negative Volumeneffekte nicht nur auf eine Solvatation, sondern auch auf eine geringe Assoziation der reinen polaren Flüssigkeit zurückzuführen.

Die Deutung erfährt eine Unterstützung durch die Bestimmung der Mischungswärme ${ }^{3}$. Diese ist im Fall Aceton-Schwefelkohlenstoff negativ und entspricht etwa der aufzuwendenden Entassoziationsenergie; im Fall Chlorbenzol-Hexan ist sie positiv und ist ein ungefähres $\mathrm{Ma}$ für die freiwerdende Solvatationswärme. Wenn es sich um eine assoziierte Substanz handelt, wäre die Mischungswärme die Differenz von Solvatations- und Entassoziationsenergie, also etwa:

$$
\underset{(\mathrm{A} B)}{Q_{\mathrm{M}} \sim \underset{(\mathrm{A}-\mathrm{A})}{<0}} \underset{\substack{Q_{\text {Sitalv }} \\(\mathrm{A}-\mathrm{B})}}{Q_{0}}
$$

Weniger einfach sind die Volumeneffekte an Gemischen polar-unpolar zu deuten, bei denen die $\Delta V$ nur kleine positive Werte annehmen. Es kann dann entweder der geometrische Effekt so klein sein, daß der größte Anteil der Volumendilatation einer Entassoziation zuzuschreiben ist; oder aber die Wechselwirkungsenergie reicht nicht zur vollständigen Entassoziation der polaren Substanz aus, um den Pakkungseffekt wesentlich zu vergrößern. Das ist z. B. der Fall, wenn Mischungslücken auftreten (HexanMethanol, $\mathrm{CS}_{2}$-Methanol bei $20^{\circ} \mathrm{C}$ ). Oder es findet eine nur geringe Solvatation der Moleküle A mit den Molekülen B statt, die den positiven, geometrischen

3 s. Physik.-chem. Taschenbuch, Mischungs- und Lösungswärmen, S. 1226, bearb. von H. H a r m s, Akad. Verl.Ges., Leipzig 1949. Dort auch ausführliche Literaturangaben. 
Volumeneffekt nur unerheblich verringert. In derartigen Fällen müssen zur Deutung des Volumeneffektes noch andere charakteristische Größen herangezogen werden.

Sehr viel unübersichtlicher sind die Verhältnisse bei Gemischen aus zwei Dipolkomponenten. Im allgemeinen sind beide als Reinsubstanzen assoziiert; im Gemisch kann eine gegenseitige, mehr oder weniger vollständige Entassoziation und die Bildung von Mischassoziation erfolgen und u. U. kann es sogar zu fester gebundenen Übermolekülen kommen. Fast immer findet man an Dipol-Dipolgemischen Volumenkontraktionen. Dann ist jedenfalls die volumenverkleinernde zwischenmolekulare Attraktion so groß, daß die volumenvergrößernden Entassoziations- und Packungseffekte überkompensiert werden.

Treten in Dipol-Dipolgemischen positive Effekte auf, so läßt sich aus dem Volumeneffekt allein keine eindeutige Aussage herausschälen.

Auch die Mischungswärmen lassen hier nur sehr bedingt Schlüsse zu. Positive Mischungswärmen bedeuten immer eine freiwerdende Bindungsenergie. Bei negativer Mischungswärme kann ebenfalls eine Bindung zwischen verschiedenartigen Molekülen stattgefunden haben, vor allem wenn die Entassoziationsenergien groß sind oder nicht alle entassoziierten Moleküle ein Mischassoziat eingehen. Nennt man $\lambda_{\mathrm{A}}$ den Bruchteil der entassoziierten Moleküle $\mathrm{A}, \lambda_{\mathrm{B}}$ denjenigen der Sorte $\mathrm{B}, x_{\mathrm{A}}$ und $x_{\mathrm{B}}$ die betreffenden Molanteile und $x$ den Molanteil der Übermoleküle aus $\mathrm{A}$ und $\mathrm{B}$, so setzt sich die Mischungswärme ungefähr folgendermaßen zusammen:

$$
\underset{(\mathrm{AB})}{Q_{\mathrm{M}}}=i_{\mathrm{A}} x_{\mathrm{A}} \underset{(\mathrm{A}-\mathrm{A})}{Q_{\text {Entass }}}+i_{\mathrm{B}} x_{\mathrm{B}} \underset{(\mathrm{B}-\mathrm{B})}{Q_{\text {Entass }}}+\cdots \underset{(\mathrm{A}-\mathrm{B})}{Q_{\mathrm{Ass}}} .
$$

Die $Q_{\text {Entass }}$ sind sicher negativ, $Q_{\text {Ass }}$ hingegen positiv, wenn Mischassoziation stattfindet. Da die Zahl der gebildeten Übermoleküle sicher kleiner oder höchstens gleich der Zahl der entassoziierten Moleküle jeder Sorte ist, so sieht man, daß es zu einer negativen Mischungswärme kommen kann, auch dann, wenn

$$
\underset{(\mathrm{A}-\mathrm{A})}{Q_{\text {Entas }}}+\underset{(\mathrm{B}-\mathrm{B})}{Q_{\text {Entass }}}<\left|\underset{(\mathrm{A}-\mathrm{B})}{Q_{\text {Ass }}}\right| \text { ist. }
$$

Bei negativen Volumeneffekten binärer Gemische polarer Substanzen kann man demnach auf die Bil1930

4 P. Debye, Polare Moleküle, Verlag Hirzel, Leipzig

5 R. M e cke u. A. Reuter u. R. L. S chupp, Z. Naturforschg. 4 a, 182 [1949].

6 R. M e cke u. A. R e u t e r, Z. Naturforschg. 4a, 368 [1949]. dung von Mischassoziation bzw. von Übermolekülen schließen, bei positiven Volumeneffekten lassen sich derartige Aussagen nicht ohne weiteres machen.

b) Das Dipolmoment

Die Dipolmomente der hier untersuchten Substanzen sind aus Messungen im Gaszustand recht genau bekannt und wurden für diesen aus der DebyeClausius - Mosottischen (DCM)-Formel bestimmt. Wendet man die DCM-Formel, die ja prinzipiell wegen der Berechnung des inneren Feldes die Voraussetzung des flüssigen Zustandes einer Substanz nicht berücksichtigt, auf Flüssigkeiten an und bestimmt die Dipolmomente polarer Substanzen durch die Verdünnungsmethode ${ }^{4}$, so erhält man zwar bei extremer Verdünnung für das Dipolmoment des einzelnen Moleküls Werte, die mit denen aus Gasmessungen $u$. U. gut übereinstimmen. Das Verhalten der Dipolteilchen bei größerer Konzentration bzw. auch die zwischenmolekularen Vorgänge, insbesondere bei Gemischen von zwei Dipolkomponenten, gibt die DCM-Formel offenbar nicht richtig wieder, wie vor allem $\mathrm{M} \mathrm{e} \mathrm{c} \mathrm{k} \mathrm{e} \mathrm{und} \mathrm{Mitarbb.} .^{5}$ gezeigt haben.

Die nach der DCM-Formel berechneten Dipolmomente zeigen - vor allem bei Lösung von polaren Substanzen in unpolaren - eine starke Konzentrationsabhängigkeit, aus deren Verlauf man auf die Assoziationsverhältnisse der Dipolmoleküle schloß. $\mathrm{Da}$ es sich dabei aber fast immer um eine Überlagerung verschiedener Assoziationszustände handelt, so mußte die Deutung oft recht willkürlich $\operatorname{sein}^{6,7}$.

Nun haben in der letzten Zeit $\mathrm{Hü} \mathrm{ckel}^{8}$ und vor allem $\mathrm{Mecke}$ und Mitarbb. eine zuerst von Onsager ${ }^{9}$ und später von Kirkwood ${ }^{10}$ u. a. entwickelte Theorie über das Dipolmoment des Flüssigkeitsmoleküls aufgegriffen und nach ihr eine Reihe von DK-Messungen ausgewertet und überprüft. Dabei hat sich gezeigt, dâß die Onsagersche Theorie, in der das innere Feld unter Berücksichtigung der Induktionswirkung der Dipole berechnet wird, offenbar den tatsächlichen Gegebenheiten besser gerecht wird als die ältere DCM-Formel, die ja ursprünglich auch nur für Gase berechnet und nicht zur Anwendung auf Flüssigkeiten gedacht war. Die normale Dipol-Dipolanziehung in der Onsagerschen Berechnung der Dipolmomente ist extrapoliert, und man

7 R. M e c k e, Z. Elektrochem. angew. physik. Chem. 52, 269 [1948].

8 W. H ü c k e l, Z. Naturforschg. 3 b, 138 [1948].

9 L. O n s a g e r, J. Amer. chem. Soc. 58, 1486 [1936].

10 J. G. K ir k w o od, J. chem. Physics 4, 592 [1936]; 7, 911 [1939]. 
sollte daher für normal assoziierende Dipolsubstanzen - gelöst in einer unpolaren Substanz - einen ungefähr konstanten, d. h. konzentrationsunabhängigen Wert für das Dipolmoment finden. Erhebliche Abweichungen hiervon dagegen zeigen, daß außer der normalen Dipolassoziation noch andere zwischenmolekulare Wechselwirkungskräfte auftreten, z. B. solche, die eine H-Brückenbildung zur Folge haben.

Handelt es sich um Gemische aus einer polaren Substanz A und einer unpolaren Substanz B, so erhält man das Dipolmoment der Moleküle der Sorte A nach Onsager:

$$
\begin{aligned}
x_{\mathrm{A}} \gamma_{\mathrm{A}}{ }^{2} \mu_{\mathrm{A}}{ }^{2}=\frac{9 k T}{4 \pi N_{\mathrm{L}}}[ & x_{\mathrm{A}} V_{\mathrm{A}} \gamma_{\mathrm{A}} \frac{\varepsilon-n_{\mathrm{A}}{ }^{2}}{n_{\mathrm{A}}{ }^{2}+2} \\
& \left.+x_{\mathrm{B}} V_{\mathrm{B}} \gamma_{\mathrm{B}} \frac{\varepsilon-n_{\mathrm{B}}{ }^{2}}{n_{\mathrm{B}}{ }^{2}+2}\right],
\end{aligned}
$$

wobei $\gamma_{\mathrm{A}}$ und $\gamma_{\mathrm{B}}$ die für das innere Feld charakteristischen Faktoren sind. Es gilt:

$$
\gamma_{\mathrm{A}}=\frac{\varepsilon\left(n_{\mathrm{A}}{ }^{2}+2\right)}{2 \varepsilon+n_{\mathrm{A}}{ }^{2}} ; \quad \gamma_{\mathrm{B}}=\frac{\varepsilon\left(n_{\mathrm{B}}{ }^{2}+2\right)}{2 i+n_{\mathrm{B}}{ }^{2}} .
$$

$\varepsilon$ ist die gemessene DK des Gemisches und $n$ der auf unendlich extrapolierte Brechungsindex unter Berücksichtigung von Elektronen- und Atompolarisation (s. u. a. M ecke und S chupp ${ }^{11}$ ), $x_{\mathrm{A}}$ und $x_{\mathrm{B}}$ sind die Molanteile der Gemischpartner (für binäre $\mathrm{Ge}$ mische: $x_{\mathrm{A}}+x_{\mathrm{B}}=1$ ).

Die Onsagersche Formel geht in die bekannte DCM-Formel über, wenn man setzt:

$$
\gamma_{\mathrm{A}}=\gamma_{\mathrm{B}}=\frac{\varepsilon+2}{3} \text {. }
$$

Handelt es sich um Gemische aus zwei polaren Flüssigkeiten, so gilt:

$$
\begin{aligned}
& x_{\mathrm{A}} \gamma_{\mathrm{A}}{ }^{2} \mu_{\mathrm{A}}{ }^{2}+x_{\mathrm{B}} \gamma_{\mathrm{B}}{ }^{2} \mu_{\mathrm{B}}{ }^{2}=\frac{9 k T}{4 \pi N_{\mathrm{L}}} \\
& \left(x_{\mathrm{A}} V_{\mathrm{A}} \gamma_{\mathrm{A}} \frac{\varepsilon-n_{\mathrm{A}}{ }^{2}}{n_{\mathrm{A}}{ }^{2}+2}+x_{\mathrm{B}} V_{\mathrm{B}} \gamma_{\mathrm{B}} \frac{\varepsilon-n_{\mathrm{B}}{ }^{2}}{n_{\mathrm{B}}{ }^{2}+2}\right)
\end{aligned}
$$

und für zwei polare Substanzen im Gemisch mit einer dritten, unpolaren:

$$
\begin{gathered}
x_{\mathrm{A}} \gamma_{\mathrm{A}}{ }^{2} \mu_{\mathrm{A}}{ }^{2}+x_{\mathrm{B}} \gamma_{\mathrm{B}}{ }^{2} \mu_{\mathrm{B}}{ }^{2}=\frac{9 k T}{4 \pi N_{\mathrm{L}}}\left(x_{\mathrm{A}} V_{\mathrm{A}} \gamma_{\mathrm{A}} \frac{\varepsilon-n_{\mathrm{A}}{ }^{2}}{n_{\mathrm{A}}{ }^{2}+2}\right. \\
\left.+x_{\mathrm{B}} V_{\mathrm{B}} \gamma_{\mathrm{B}} \frac{\varepsilon-n_{\mathrm{B}}{ }^{2}}{n_{\mathrm{B}}{ }^{2}+2}+x_{\mathrm{C}} V_{\mathrm{C}} \gamma_{\mathrm{C}} \frac{\varepsilon-n_{\mathrm{C}}{ }^{2}}{n_{\mathrm{C}}{ }^{2}+2}\right) .
\end{gathered}
$$

11 R. L. S chupp u. M. Mecke, Z. Elektrochem. angew. physik. Chem. 52, 54 [1948].
Insbesondere die beiden zuletzt angegebenen Formeln bedürfen noch einer gesonderten Diskussion:

Grundsätzlich ist zunächst nichts darüber bekannt, in welcher Weise die beiden polaren Flüssigkeiten untereinander reagieren. Will man das Verhalten der Dipolmomente der Substanz A bestimmen, so müßte bekannt sein, ob und in welcher Weise die Dipole der Substanz B von denen der Substanz A beeinflußt werden. Diese Aussagen zugleich liefert keine der beiden Gleichungen. Daher wurde ein mittleres Momentquadrat definiert, das von einem Moment herrühren soll, das sich durch die Wechselwirkung der beiden Substanzen im Mittel pro Molekül ergibt. Dabei kann es sich um Moleküle beider Substanzsorten mit je nach der Konzentration verschiedener mittlerer Zähligkeit handeln, und es bleibt zunächst offen, ob neben einer Mischung der Sorten A und B mit normaler Assoziation, durch eine Kopplung der Dipolmomente $\mu_{\mathrm{A}}$ und $\mu_{\mathrm{B}}$ Mischmoleküle entstehen, deren Bindung auf andere Effekte als die normale Dipol-Dipolanziehung zurückgeht.

Die entsprechende Definitionsgleichung lautet:

$$
x_{\mathrm{A}} \gamma_{\mathrm{A}}{ }^{2} \mu_{\mathrm{A}}{ }^{2}+x_{\mathrm{B}} \gamma_{\mathrm{B}}{ }^{2} \mu_{\mathrm{B}}{ }^{2}=\left(x_{\mathrm{A}}+x_{\mathrm{B}}\right) \overline{\gamma^{2}} \overline{\mu^{2}},
$$

wobei sich bei der Berechnung zeigte, daß man für die später aufgezeigten Fälle $\overline{\mu^{2}}=\bar{\mu}^{2}$ setzen kann. $\bar{\gamma}$ wird definiert durch:

$$
\begin{aligned}
& \frac{n^{2}-1}{n^{2}+2} \bar{\gamma} V=\frac{n_{\mathrm{A}}{ }^{2}-1}{n_{\mathrm{A}}{ }^{2}+2} \gamma_{\mathrm{A}} x_{\mathrm{A}} V_{\mathrm{A}} \\
& \quad+\frac{n_{\mathrm{B}}{ }^{2}-1}{n_{\mathrm{B}}{ }^{2}-2} \gamma_{\mathrm{B}} x_{\mathrm{B}} V_{\mathrm{B}},
\end{aligned}
$$

wobei

$$
\bar{\gamma}=\frac{\varepsilon\left(n^{2}+2\right)}{2 \varepsilon+n^{2}} ; \quad V=x_{\mathrm{A}} V_{\mathrm{A}}+x_{\mathrm{B}} V_{\mathrm{B}}
$$

(In den angegebenen Formeln ist der Volumeneffekt nicht enthalten, da er keine sehr wesentliche Rolle für die Größe der Dipolmomente spielt. In der späteren zahlenmäßigen Berechnung wurde er berücksichtigt, indem er in Gemischen polar-unpolar dem polaren Gemischpartner zugeschrieben wurde. Bei Anwesenheit zweier polarer Komponenten wurde er im Verhältnis ihrer Volumenanteile aufgeteilt.) Die $\bar{\mu}$-Werte lassen sich also aus der DK der Gemische berechnen. Es kann sich nun in Gemischen aus den beiden polaren Partnern A und B bei der Substanz A um Dipole handeln, für die durch DKMessungen an Gemischen der Substanz A mit einer unpolaren Substanz C eine normale Assoziation, für 
die Substanz B hingegen in Gemischen mit C eine Brückenbildung festgestellt wurde, wie sie von $\mathrm{Mecke}^{6}$ für Phenol in Tetrachlorkohlenstoff gezeigt wird. Dann kann man nach einem Vorschlag von Mecke näherungsweise für die Substanz A das aus den Messungen in unpolarer Substanz bestimmte, nur unwesentlich konzentrationsabhängige $\mu^{0} \mathrm{~A}$ setzen und erhält dann für $\mu_{\mathrm{B}}$ den die Wechselwirkung mit $\mu_{\mathrm{A}}$ enthaltenden Wert: $\mu_{\mathrm{B}(\mathrm{A})}$.

Man kann dann formulieren:

$$
\begin{array}{r}
\left(x_{\mathrm{A}}+x_{\mathrm{B}}\right) \bar{\gamma}^{2} \bar{\mu}^{2}=x_{\mathrm{A}} \gamma_{\mathrm{A}}{ }^{2} \mu_{\mathrm{A}}{ }^{2}+x_{\mathrm{B}} \gamma_{\mathrm{B}}{ }^{2} \mu_{\mathrm{B}}{ }^{2} \\
=x_{\mathrm{A}} \gamma_{\mathrm{A}}{ }^{2} \mu_{\mathrm{A}}{ }^{0^{2}}+x_{\mathrm{B}} \gamma_{\mathrm{B}}{ }^{2} \mu_{\mathrm{B}(\mathrm{A})}{ }^{2} .
\end{array}
$$

Der Wert $\mu_{\mathrm{B}(\mathrm{A})}{ }^{2}$ ergibt sich dann zu:

$\mu_{\mathrm{B}(\mathrm{A})}{ }^{2}=\frac{1}{x_{\mathrm{B}} \gamma_{\mathrm{B}}{ }^{2}}\left[\left(x_{\mathrm{A}}+x_{\mathrm{B}}\right) \bar{\gamma}^{2} \bar{\mu}^{2}-x_{\mathrm{A}} \gamma_{\mathrm{A}}{ }^{2} \mu_{\mathrm{A}^{\nu^{2}}}\right]$.

Das gilt auch für den Fall zweier Dipolsubstanzen im Gemisch mit einer unpolaren Substanz $\left(x_{\mathrm{A}}+x_{\mathrm{B}}<1\right)$. Es wird sich zeigen, daß die analogen Überlegungen für die DCM-Formel nicht durchführbar sind.

Bestimmung der Konzentrationsabhängigkeit der Volumeneffekte und der Dipolmomente

Die DK- und $\Delta V$-Werte wurden an folgenden Reinsubstanzen bzw. Cemischen durchgeführt:

1. Chlorbenzol (Cbz), Methanol (M) und Hexan $(\mathrm{H})$ :

a) binäre Gemische,

b) ternäre Gemische aus Methanol in Hexan mit den Molanteilen $\frac{x_{\mathrm{M}}}{x_{\mathrm{M}}+x_{\mathrm{H}}}=0,9272$ mit verschiedenen Zusätzen von Chlorbenzol.

2. Aceton (Ac), Methanol (M), Schwefelkohlenstoff $(\mathrm{C})$ :

a) binäre Gemische,

b) ternäre Gemische aus Methanol mit Schwefelkohlenstoff mit den Molanteilen $\frac{x_{M}}{x_{M}+x_{C}}=0,8551$ mit Zusatz von Aceton.

Die Substanzen wurden von Merck bzw. S,cheringKahlbaum12 bezogen und mit Hilfe üblicher Destillations- und Trocknungsverfahren vorbehandelt. Die DK wurden mit einer Überlagerungsmethode bei einer Frequenz von $10^{6} \mathrm{~Hz}$ bestimmt und zur Dichtemessung

12 „Hexan aus Petroleum“, Schering-Kahlbaum; Schwefelkohlenstoff ,reinst“, Schering-Kahlbaum; Methanol p.A., Schering-Kahlbaum; Aceton, reinst, Merck; Monochlorbenzol, I.G. Ludwigshafen. wurde eine Mohrsche Waage benutzt. Sämtliche Messungen erfolgten bei $20^{\circ} \mathrm{C}$. Die Flüssigkeiten befanden sich bei jeder Messung in wasserumflossenen Gefäßen, die an einen Thermostaten angeschlossen waren. Die Mischungen wurden eingewogen. Es muß hervorgehoben werden, daß sowohl das System Methanol-Schwefelkohlenstoff wie auch das System Methanol-Hexan bei Zimmertemperatur. eine erhebliche Mischungslücke aufweisen.

In den graphischen Darstellungen und Tabellen sind für die beiden Systeme die aus den Dichtebestimmungen berechneten Volumeneffekte und die aus den DK-Messungen nach der DCM-Formel und der Onsagerschen Formel erhaltenen Dipolmomente angegeben; dabei haben die $\bar{u}$ der Gemische mit zwei Dipolpartnern die durch (Gl. 9) definierte Bedeutung.

\section{Diskussion der Meßergebnisse}

\section{Hexan-Methanol-Chlorbenzol}

Wegen der bei $20^{\circ} \mathrm{C}$ breiten Mischungslücke sind nur wenige Zusammensetzungen des Systems MethanolHexan untersucht. Aber es zeigt sich, daß diese Gemische einen positiven Volumeneffekt haben (Abb. 1 a und Tab. 1 a), der auf geometrische Ursachen und teilweise Entassoziation zurückgehen kann. Das nach der DCM-Formel berechnete Dipolmoment fällt $a b$ und hat für reines Methanol den kleinsten Wert. Dazwischen müßte ohne Existenz der Mischungslücke ein Maximum liegen. Damit zeigt sich der für Alkohole typische Kurvenverlauf an, der bisher zu verhältnismäßig willkürlichen Annahmen über die Alkohol-Assoziation geführt hat. Nach der Onsagerschen Formel aber hat der unverdünnte Alkohol ähnlich wie das von Mecke untersuchte Phenol - das größte Dipolmoment. Der Anstieg wird durch zwischenmolekulare Kräfte besonderer Art gedeutet; denn für eine normale Dipolassoziation müßte sich nach der Onsagerschen Theorie ein ungefähr konstanter, konzentrationsunabhängiger Wert des Dipolmoments ergeben. Aus Röntgenstrukturmessungen ist bekannt, daß Alkoholmoleküle über H-Brücken zu Ketten assoziiert sind; dies verursacht den Anstieg der Onsagerschen Kurve. Bei kleinsten Konzentra-

\begin{tabular}{|c|c|c|c|c|}
\hline$x_{\mathrm{M}}$ & $\Delta V_{\mathrm{cm}^{3}}$ & $\varepsilon$ & $\mu_{\mathrm{DCM}}$ & $\mu_{\text {ons }}$ \\
\hline 0 & 0 & 1,928 & $-\overline{1}$ & $-\overline{2}$ \\
0,9272 & 0,139 & 27,6 & 1,27 & 2,95 \\
1,0 & 0 & 34,5 & 1,16 & 2,97 \\
\hline
\end{tabular}

Tab. 1a. Methanol in Hexan: $\left(x_{M}+x_{H}=1\right)$

$$
\begin{aligned}
& V_{\mathrm{M}}^{20^{\circ}}=40,455 \mathrm{~cm}^{3} ; n_{\mathrm{M}}^{2}=1,818 \\
& V_{\mathrm{H}}^{20^{\circ}}=123,262 \mathrm{~cm}^{3} ; n_{\mathrm{H}}^{2}=1,928
\end{aligned}
$$




\begin{tabular}{|c|c|c|c|c|}
\hline$x_{\mathrm{Cbz}}$ & $\Delta V_{\mathrm{cm}^{3}}$ & $\varepsilon$ & $\mu_{\mathrm{DCM}}$ & $\mu_{\mathrm{ons}}$ \\
\hline 0,9186 & $-0,205$ & 5,35 & 1,21 & 1,40 \\
0,3158 & $-0,320$ & 2,88 & 1,47 & 1,46 \\
0,2246 & $-0,260$ & $2,56_{6}$ & 1,49 & 1,46 \\
0,4318 & $-0,376$ & 3,29 & 1,42 & 1,45 \\
0,9597 & $-0,130$ & 5,55 & 1,19 & 1,40 \\
0,0347 & $-0,064$ & 2,017 & 1,55 & 1,49 \\
0.5483 & $-0,413$ & 3,73 & 1,37 & 1,43 \\
0,7232 & $-0,407$ & 4,45 & 1,30 & 1,41 \\
0,7828 & $-0,382$ & 4,72 & 1,27 & 1,41 \\
0,5842 & $-0,418$ & 3,87 & 1,35 & 1,43 \\
1,0 & 0 & 5,74 & 1,18 & 1,39 \\
0 & 0 & 1,928 & 0 & 0 \\
\hline
\end{tabular}

Tab. 1b. Chlorbenzol in Hexan: $\left(x_{\mathrm{Cbz}}+x_{\mathrm{H}}=1\right)$ $V_{\mathrm{Cbz}}^{20^{\circ}}=101,709 \mathrm{~cm}^{3} ; n_{\mathrm{Cbz}}^{2}=2,454$
$V_{\mathrm{H}}^{20^{\circ}}=123,262 \mathrm{~cm}^{3} ; n_{\mathrm{H}}^{2}=1,928$

a)
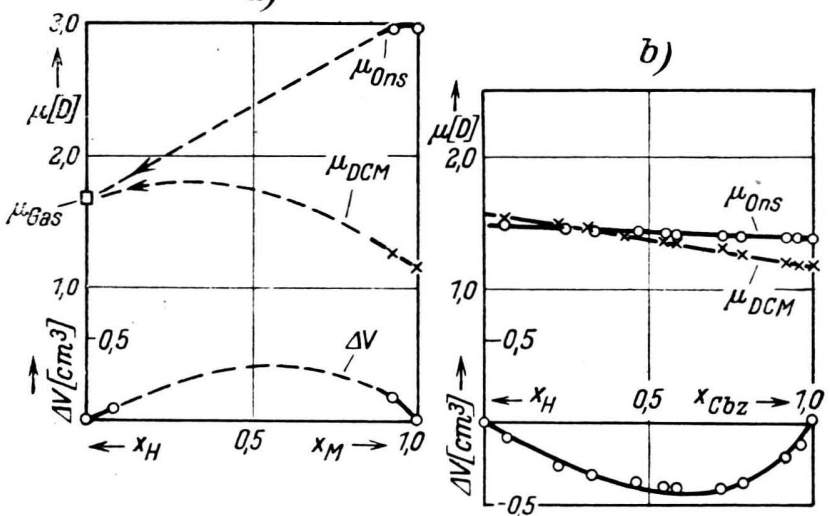

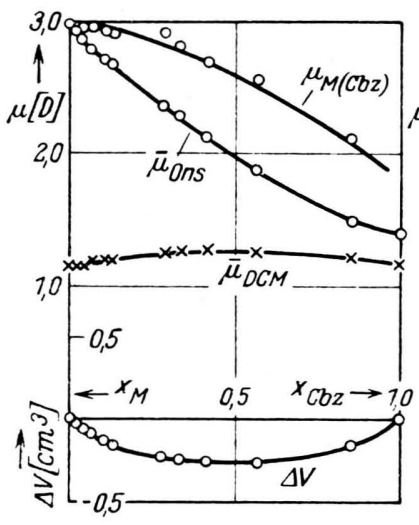

c)

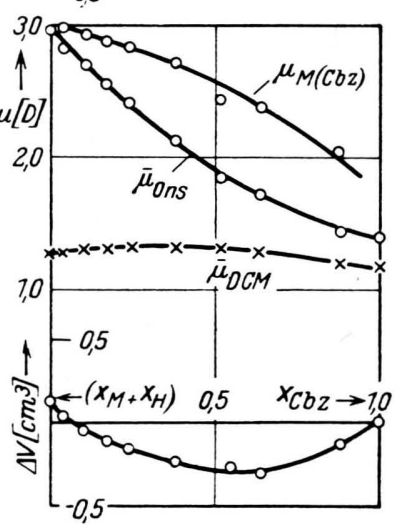

d)
Abb. 1. Konzentrationsabhängigkeit des Volumeneffekts $\Delta V\left[\mathrm{~cm}^{3}\right]$ und des Dipolmoments $\mu$ [D] für die Gemische: a) Methylalkohol $\left[\mathrm{CH}_{3} \mathrm{OH}\right](\mathrm{M})$ in Hexan $\left[\mathrm{C}_{6} \mathrm{H}_{14}\right](\mathrm{H})$; b) Chlorbenzol $\left[\mathrm{C}_{6} \mathrm{H}_{5} \mathrm{Cl}\right](\mathrm{Cbz})$ in Hexan; c) Chlorbenzol in Methylalkohol; d) Chlorbenzol in Methylalkohol mit Hexan $\frac{x_{\mathrm{M}}}{x_{\mathrm{M}}+x_{\mathrm{H}}}=0,9272$. (Temperatur $20^{\circ} \mathrm{C}$.) ----Mischungslücke. (Für $\Delta V$ gilt der innere, für $\mu$ der äußere Ordinatenmaßstab.)

\begin{tabular}{|c|c|c|c|c|c|}
\hline$x_{\mathrm{Cbz}}$ & $\Delta V_{\mathrm{cm}^{3}}$ & $\varepsilon$ & $\bar{\mu}_{\mathrm{DCM}}$ & $\bar{\mu}_{\mathrm{ons}}$ & $\mu_{\mathrm{M}(\mathrm{Cbz}) \text { ons }}$ \\
\hline 0,4159 & $-0,253$ & 14,20 & $1,28_{3}$ & $2,12_{5}$ & 2,73 \\
0,5668 & $-0,258$ & 10,35 & $1,27_{4}$ & $1,88_{6}$ & 2,58 \\
0,3295 & $-0,240$ & 17,14 & $1,27_{4}$ & $2,29_{3}$ & 2,82 \\
0,2867 & $-0,233$ & 18,70 & $1,26_{6}$ & $2,37_{0}$ & 2,91 \\
0,8561 & $-0,158$ & 6,52 & $1,20_{2}$ & $1,48_{3}$ & 2,11 \\
0,1290 & $-0,151$ & 26,09 & $1,21_{8}$ & $2,68_{0}$ & 2,90 \\
0,1036 & $-0,130$ & 27,44 & $1,20_{8}$ & $2,72_{6}$ & 2,93 \\
0,0165 & $-0,020$ & 32,89 & $1,16_{9}$ & $2,91_{6}$ & 2,90 \\
0,0352 & $-0,050$ & 31,2 & $1,17_{5}$ & $2,85_{8}$ & 2,93 \\
0,0663 & $-0,088$ & 29,5 & $1,19_{1}$ & $2,80_{1}$ & 2,91 \\
0,0 & 0 & 34,5 & $1,16_{0}$ & $2,97_{0}$ & 2,97 \\
1,0 & 0 & 5,74 & $1,17_{5}$ & $1,39_{3}$ & 0 \\
\hline
\end{tabular}

Tab. 1c. Chlorbenzol in Methanol: $\left(x_{\mathrm{Cbz}}+x_{\mathrm{M}}=1\right)$

$$
\begin{aligned}
& V_{\mathrm{M}}=40,455 \mathrm{~cm}^{3} ; n^{2}=1,818 \\
& V_{\mathrm{Cbz}}=101,709 \mathrm{~cm}^{3} ; n^{2}=2,454
\end{aligned}
$$

tionen existieren nur Einermoleküle, während in rei- , nem Methanol Ketten verschiedener Länge vorliegen.

Das Dipolmoment von Chlorbenzol in Hexan (Abb. $1 \mathrm{~b}$ und Tab. 1b) nimmt, nach der DCM-Formel berechnet, mit steigender Konzentration ab, nach Onsager berechnet zeigt es eine nur sehr geringe Abnahme und ist im Vergleich zu Methylalkohol als fast konstant anzusehen. Dies deutet nach Onsager auf eine Assoziation durch Dipol-Dipolanziehung hin.

Der Volumeneffekt von Hexan-Chlorbenzol-Gemischen ist negativ. Man kann hieraus schließen, daß Chlorbenzol, das auf Grund seiner DCM-Kurve des Dipolmoments nur als schwach assoziiert anzusehen ist, durch Hexan entassoziiert wird und darauf mit den Hexan-Molekülen Solvatationskomplexe bildet, die eine so große molare Volumenkontraktion hervorrufen, daß die durch Packungseffekt und Entassoziation hervorgerufene Dilatation überkompensiert wird.

Auch der Volumeneffekt von Methanol-Chlorbenzol-Gemischen (Abb. 1c und Tab. 1c) ist negativ. Er ist auf die Dipol-Dipolattraktion zwischen den beiden Molekülsorten zurückzuführen. In der Berechnung nach Onsager nimmt das mittlere Dipolmoment von Methanol zu Chlorbenzol ab. Nun treten aber sicher Moleküle verschiedenster Art auf; zunächst Alkoholmoleküle verschiedener Zähligkeit, gleichzeitig, aber je nach der Zusammensetzung einzelne und assoziierte Chlorbenzolmoleküle, und schließlich, denn sonst wäre ein negativer Volumeneffekt nicht möglich, mehr oder weniger fest verkettete Assoziationskomplexe Chlorbenzol-Methanol. Von allen diesen Molekülen ist $\bar{\mu}$ das sich im Mittel pro Molekül einstellende Dipolmoment. Ob nur normale Dipol- 


\begin{tabular}{|l|c|c|c|c|c|c|}
\hline$x_{\mathrm{Cbz}}$ & $x_{\mathrm{M}}$ & $\Delta V_{\mathrm{cm}^{3}}$ & $\varepsilon$ & $\bar{\mu}_{\mathrm{DCM}}$ & $\bar{\mu}_{\mathrm{ons}}$ & $\mu_{\mathrm{M}(\mathrm{Cbz}) \text { ons }}$ \\
\hline 0 & 0,9272 & $+0,139$ & 27,6 & $1,27_{4}$ & $2,95_{0}$ & 2,95 \\
0,5177 & 0,4472 & $-0,285$ & 10,4 & $1,30_{6}$ & $1,84_{3}$ & 2,44 \\
0,1033 & 0,8314 & $-0,048$ & 22,9 & $1,30_{1}$ & $2,70_{1}$ & 2,93 \\
0,6366 & 0,3369 & $-0,300$ & 8,46 & $1,27_{4}$ & $1,71_{7}$ & 2,39 \\
0,0413 & 0,8889 & $+0,045$ & 25,55 & $1,28_{6}$ & $2,84_{4}$ & 2,99 \\
0,8826 & 0,1089 & $-0,120$ & 6,17 & $1,19_{3}$ & $1,44_{3}$ & 2,06 \\
0,3825 & 0,5725 & $-0,235$ & 13,8 & $1,31_{6}$ & $2,14_{0}$ & 2,71 \\
0,2379 & 0,7066 & $-0,158$ & 17,65 & $1,32_{3}$ & $2,42_{0}$ & 2,83 \\
0,1690 & 0,7705 & $-0,105$ & 20,02 & $1,31_{2}$ & $2,55_{1}$ & 2,87 \\
1,0 & 0 & 0 & 5,74 & $1,17_{5}$ & $1,39_{3}$ & \\
\hline
\end{tabular}

Tab. 1d. Chlorbenzol in Methanol mit Hexan:

$$
\begin{gathered}
\frac{x_{\mathrm{M}}}{x_{\mathrm{M}}+x_{\mathrm{H}}}=0,9272 \\
\left(x_{\mathrm{Cbz}}+x_{\mathrm{M}}+x_{\mathrm{H}}=1\right) \\
V_{\mathrm{M}}^{20^{\circ}}=40,455 \mathrm{~cm}^{3} ; n_{\mathrm{M}}^{2}=1,818 \\
V_{\mathrm{H}}^{20^{\circ}}=123,262 \mathrm{~cm}^{3} ; n_{\mathrm{H}}^{2}=1,928 \\
V_{\mathrm{Cbz}}^{20^{\circ}}=101,709 \mathrm{~cm}^{3} ; n_{\mathrm{Cbz}}^{2}=2,454
\end{gathered}
$$

Dipolanziehung zwischen den beiden Molekülarten oder ob spezifische Wechselwirkungskräfte vorhanden sind, kann man feststellen, wenn man nach Onsager unter Verwendung des Moments von reinem Chlorbenzol $\left(\mu_{0}=1,4 \mathrm{D}, \mathrm{u}\right.$. U. auch ein konzentrationsabhängiges, wie es sich z. B. für Chlorbenzol in Hexan ergibt) das Moment von Methylalkohol berechnet, dem man dann etwa auftretende spezifische Kräfte zuordnen würde. Es ergibt sich dieses Moment von Methanol in Chlorbenzol (s. a. Abb. 1 c) aus:

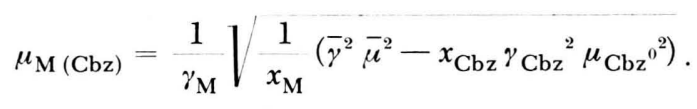

Vergleicht man dieses Moment von Methanol mit dem in Mischung mit Hexan bzw. Schwefelkohlenstoff (Abb. 2) erhaltenen, so hat es bis auf die in Chlorbenzol nicht auftretende Mischungslücke fast den gleichen konzentrationsabhängigen Verlauf. Demnach verhält sich Methylalkohol in Chlorbenzol nicht wesentlich anders als in einer unpolaren Substanz. Es wird lediglich eine Dipol-Dipolanziehung zwischen den beiden Molekülsorten auftreten; spezifische Wechselwirkungskräfte zwischen Chlorbenzol und Methanol existieren offenbar nicht; sehr wahrscheinlich werden die H-Brücken des Methanols durch Verdünnung mit Chlorbenzol in ungefähr dem gleichen Maße zerstört wie durch eine unpolare Substanz.

Die analogen Überlegungen, um auch aus den nach der DCM-Formel berechneten $\bar{\mu}$-Werten das Chlorbenzolmoment zu extrapolieren, sind grundsätzlich nicht möglich, denn in den berechneten Werten sind sämtliche zwischenmolekularen Effekte, also auch die einfache Dipolassoziation, implizit enthalten. Daher sind dort immer, auch wenn es sich nicht um eine H-Brückenbildung handelt, die Dipolmomente sehr stark konzentrationsabhängig, und man kann daher auch nicht näherungsweise, hier z. B. durch Verwendung des Chlorbenzolmoments, zu einem sinnvollen Wert für den 2. Dipolpartner kommen, wie sich auch durch einfache Rechnung zeigen läßt.

Setzt man nun einem in molaren Anteilen festgelegten Gemisch Hexan in Methanol: $\frac{x_{\mathrm{M}}}{x_{\mathrm{M}}+x_{\mathrm{H}}}=0,9272$, als dritte Substanz Chlorbenzol hinzu (Abb. 1 d und Tab. 1d), so wird die Volumenkontraktion noch etwas größer als bei Chlorbenzol-Methanol-Gemischen. Der Grund dafür dürfte darin liegen, daß erstens Chlorbenzol mit Hexan solvatisiert und zweitens Chlorbenzol mit Methanol assoziiert, was beides zu einem negativen Volumeneffekt führt. Offenbar wird die volumenvergrößernde Entassoziation von Methanol durch die zunehmende Zahl der mit Chlorbenzol assoziationsfähigen Einermoleküle kompensiert.

Der Verlauf des konzentrationsabhängigen Dipolmoments ist ganz ähnlich wie im binären Gemisch Methanol-Chlorbenzol; die nach der DCM-Formel berechneten $\bar{\mu}$-Werte liegen etwas höher und die nach Onsager berechneten etwas tiefer als für das binäre Gemisch. Nimmt man wieder ein konstantes Moment für Chlorbenzol an $\left(\mu_{\mathrm{Cbz}}=1,4 \mathrm{D}\right)$, so ergibt sich aus Gl. 13 für Methanol genau das gleiche Dipolmoment wie für das binäre Gemisch. Chlorbenzol hat also hinsichtlich der die Alkohol-Kettenbildung betreffenden zwischenmolekularen Kräfte offenbar die gleiche Wirkung wie Hexan, da der Verlauf von $\mu_{\mathrm{M}(\mathrm{Cbz})}$ unabhängig davon ist, ob Methanol in Hexan (bis auf die nicht zugängliche Mischungslücke) oder in Chlorbenzol oder in beiden gelöst ist. Diese Tatsache erhärtet die Annahme, daß Chlorbenzol selbst nur schwach assoziiert und daher besonders zur Solvatation mit unpolaren Substanzen geneigt ist, was auch die negativen Volumeneffekte und positiven Mischungswärmen im Gemisch mit anderen unpolaren Substanzen (Dioxan, $p$-Xylol) anzeigen.

2. Aceton-Methylalkohol-Schwefelkohlenstoff

Betrachtet man zunächst die zu Hexan-Methanol analogen Gemische aus Schwefelkohlenstoff und 


\begin{tabular}{|l|c|c|c|c|}
\hline \multicolumn{1}{|c|}{$x_{\mathrm{M}}$} & $د V_{\mathrm{cm}^{3}}$ & $i$ & $\mu_{\mathrm{DCM}}$ & $\mu_{\mathrm{ons}}$ \\
\hline 0,9789 & $+0,080$ & 33,6 & 1,18 & 2,98 \\
0,0728 & $+0,041$ & 3,3 & 1,78 & 2,02 \\
0,8874 & $+0,345$ & 29,6 & 1,24 & 3,00 \\
0,7804 & $+0,447$ & 25,0 & 1,32 & 2,99 \\
0,9358 & $+0,194$ & 31,8 & 1,21 & 3,00 \\
0,8142 & $+0,420$ & 26,4 & 1,29 & 2,99 \\
0,7633 & $+0,462$ & 24,24 & 1,34 & 2,98 \\
0,0 & 0 & 2,68 & & 2,97 \\
1,0 & 0 & 34,5 & 1,16 & 2,97 \\
\hline
\end{tabular}

Tab. 2a. Methanol in Schwefelkohlenstoff: $\left(x_{\mathrm{M}}+x_{\mathrm{C}}=1\right)$

$$
\begin{aligned}
& \mathrm{V}_{\mathrm{M}}^{20^{\circ}}=40,455 \mathrm{~cm}^{3} ; n_{\mathrm{M}}^{2}=1,818 \\
& V_{\mathrm{C}}^{2 \nu^{\circ}}=60,127 \mathrm{~cm}^{3} ; n_{\mathrm{C}}^{2}=2,68
\end{aligned}
$$

a)
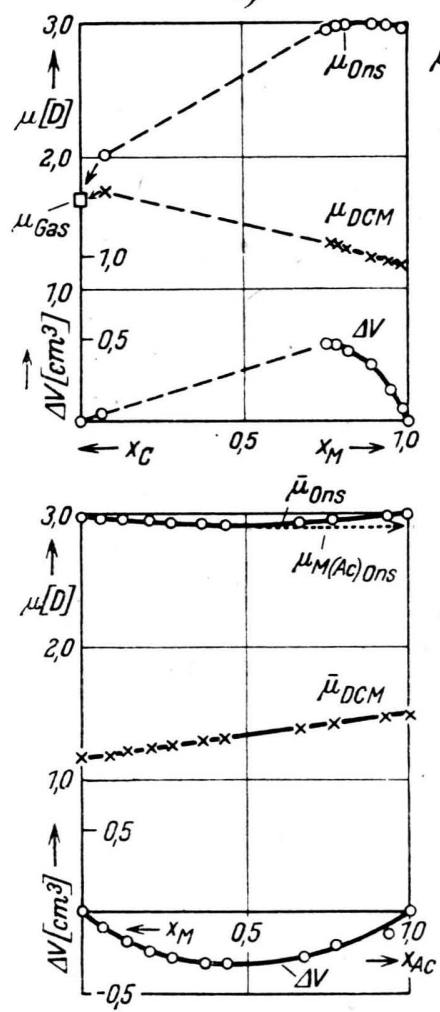

c) b)
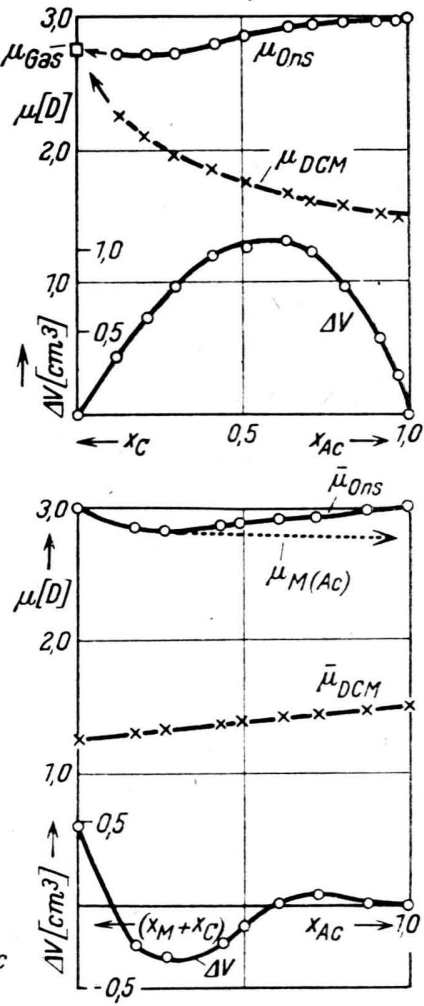

d)
Abb. 2. Konzentrationsabhängigkeit des Volumeneffekts $\Delta V\left[\mathrm{~cm}^{3}\right]$ und des Dipolmoments $\mu[D]$ für die Gemische: a) Methylalkohol $\left[\mathrm{CH}_{3} \mathrm{OH}\right](\mathrm{M})$ in Schwefelkohlenstoff $\left[\mathrm{CS}_{2}\right](\mathrm{C})$; b) Aceton $\left[\left(\mathrm{CH}_{3}\right)_{2} \mathrm{C}=\mathrm{O}\right](\mathrm{Ac})$ in Schwefe]kohlenstoff; c) Aceton in Methylalkohol; d) Aceton in Methylalkohol mit Schwefelkohlenstoff $\left(\frac{x_{\mathrm{M}}}{x_{\mathrm{M}}+x_{\mathrm{C}}}\right)=0,8551$

(Temperatur $20^{\circ}$ C.) ---- Mischungslücke. (Für $\Delta V$ gilt der innere, für $\mu$ der äußere Ordinatenmaßstab.)

\begin{tabular}{|c|c|c|c|c|}
\hline$x_{\mathrm{Ac}}$ & $د V_{\mathrm{cm}^{3}}$ & $\varepsilon$ & $\mu_{\mathrm{DCM}}$ & $\mu_{\text {ons }}$ \\
\hline 0,8034 & $+0,764$ & 17,9 & 1,56 & 2,96 \\
0,9193 & $+0,454$ & 20,1 & 1,51 & 2,97 \\
0,7071 & $+0,997$ & $16,0_{2}$ & 1,62 & 2,93 \\
0,6397 & $+1,052$ & 14,7 & 1,66 & 2,92 \\
0,5103 & $+1,006$ & $11,9_{5}$ & 1,75 & 2,85 \\
0,2913 & $+0,770$ & 7,52 & 1,96 & $2,71_{4}$ \\
0,4059 & $+0,965$ & 9,88 & 1,85 & $2,80_{7}$ \\
0,2017 & $+0,582$ & 6,10 & 2,10 & 2,74 \\
0,9673 & $+0,233$ & 21,0 & 1,49 & 2,98 \\
0,1211 & $+0,346$ & 4,67 & $2,24_{5}$ & 2,71 \\
0,0 & 0 & 2,68 & $\overline{-}$ & $-\overline{1}$ \\
1,0 & 0 & 21,8 & 1,47 & 3,0 \\
\hline
\end{tabular}

Tab. 2b. Aceton in Schwefelkohlenstoff:

$$
\begin{gathered}
\left(x_{\mathrm{Ac}}+x_{\mathrm{C}}=1\right) \\
V_{\mathrm{Ac}}^{20^{\circ}}=73,269 \mathrm{~cm}^{3} ; \quad n_{\mathrm{Ac}}^{2}=2,006 \\
V_{\mathrm{C}}=60,127 \mathrm{~cm}^{3} ; \quad n_{\mathrm{C}}^{2}=2,68
\end{gathered}
$$

Methanol (Abb. $2 \mathrm{a}$ und Tab. $2 \mathrm{a}$ ), so ist das Gesamtbild dem ersteren sehr ähnlich. Zwischen beiden Komponenten existiert bei $20^{\circ} \mathrm{C}$ eine verhältnismäßig breite Mischungslücke, die auf die zurücktretenden Attraktionskräfte gegenüber den Fremdmolekülen hinweisen. Außerhalb der Mischungslücke ist der Volumeneffekt positiv, der aus einem Packungseffekt und einer teilweisen Entassoziation resultiert, wie man aus der Konzentrationsabhängigkeit des Dipolmoments erkennt. Nach dem aus der DCM-Formel berechneten Verlauf nimmt das Dipolmoment mit abnehmender Konzentration zu, was bisher als Entassoziation gedeutet worden wäre. Die nach Onsager berechneten $\mu$-Werte nehmen, wie bei Hexan-Methanol, mit abnehmender Konzentration ab. Das bedeutet auch hier eine seltener werdende Anzahl der zwischenmolekularen H-Brücken.

Die Gemische Aceton-Schwefelkohlenstoff (Abb. $2 \mathrm{~b}$ und Tab. $2 \mathrm{~b}$ ) haben, verglichen mit den bisherigen Systemen, einen sehr großen positiven Volumeneffekt. Man ist, da die Packungseffekte unpolarer Substanzen sehr viel kleiner sind, gezwungen, eine Entassoziation des Acetons anzunehmen, und zwar wegen der Größe des Effektes eine sehr viel vollständigere als an Methanol in $\mathrm{CS}_{2}$. Allein daraus läßt sich - was ja sonst bereits allgemein bekannt ist - für Aceton eine grundsätzlich andere Art der Assoziation herleiten, als sie für Methanol besteht. Nach der DCM-Formel berechnet sich der bekannte, mit steigender Aceton-Konzentration abnehmende Verlauf des Dipolmomentes, der ebenfalls als Assoziation gedeutet wird. Nach Onsager extrapoliert man für extreme Verdünnung etwa den gleichen Wert 


\begin{tabular}{|c|c|c|c|c|c|}
\hline$x_{\mathrm{Ac}}$ & $\Delta V_{\mathrm{cm}^{3}}$ & $\varepsilon$ & $\mu_{\mathrm{DCM}}$ & $\mu_{\text {ons }}$ & $\mu_{\mathrm{M}(\mathrm{Ac}) \mathrm{ons}}$ \\
\hline 0,3716 & $-0,311$ & 27,7 & 1,28 & 2,94 & 2,93 \\
0,1305 & $-0,191$ & 31,6 & 1,21 & 2,95 & 2,96 \\
0,6681 & $-0,267$ & $24,1_{5}$ & 1,38 & 2,94 & 2,92 \\
0,7633 & $-0,206$ & $23,4_{4}$ & 1,40 & 2,96 & 2,90 \\
0,9285 & $-0,152$ & $22,3_{3}$ & 1,45 & 2,99 & 2,91 \\
0,4355 & $-0,307$ & $26,7_{8}$ & 1,30 & 2,94 & 2,92 \\
0,2707 & $-0,291$ & $29,2_{0}$ & 1,25 & 2,94 & 2,94 \\
0,2031 & $-0,243$ & $30,2_{4}$ & 1,23 & 2,94 & 2,95 \\
0,0569 & $-0,096$ & $33,1_{5}$ & 1,18 & 2,96 & 2,97 \\
1,0 & 0 & 21,8 & 1,47 & 3,00 & 3,00 \\
0,0 & 0 & 34,5 & 1,16 & 2,97 & 2,97 \\
\hline
\end{tabular}

Tab. 2c. Aceton in Methanol $\left(x+x_{M}=1\right)$

$$
\begin{aligned}
& V_{\mathrm{M}}^{20^{\circ}}=40,455 \mathrm{~cm}^{3} ; n_{\mathrm{M}}^{2}=1,818 \\
& V_{\mathrm{Ac}}^{20^{\circ}}=73,269 \mathrm{~cm}^{3} ; n_{\mathrm{Ac}}^{2}=2,006
\end{aligned}
$$

$\left(\mu_{\mathrm{Ac}} \sim 2,75 \mathrm{D}\right)$. Für zunehmende Konzentration steigt dieser Wert um etwas weniger als $10 \%$ für reines Aceton an $\left(\mu_{\mathrm{Ac}} \sim 3,0 \mathrm{D}\right)$. - Einen ganz ähnlichen Verlauf findet R. Mecke (1. c. ${ }^{7}$, S. 280) für Aceton in Tetrachlorkohlenstoff: In erster Näherung ist das nach Onsager berechnete Dipolmoment von Aceton als konstant anzusehen; man muß annehmen, daß seine Assoziation im wesentlichen auf Dipol-Dipolanziehung beruht ${ }^{6}$.

Der positive Volumeneffekt von Aceton in $\mathrm{CS}_{2}$ ist also aus der Konzentrationsabhängigkeit des Dipolmoments nach der Debyeschen als auch nach der Onsagerschen Theorie zu verstehen. Jedoch nur die Dipolmomente $\mu_{\mathrm{DCM}}$ spiegeln die dem positiven Volumeneffekt zugrundeliegende Entassoziation direkt wider. Da die Aceton-Assoziation im wesentlichen nur auf Dipol-Dipolanziehung beruht, kann sie voraussetzungsgemäß in den Momenten $\mu_{\text {ons }}$ nicht zum Ausdruck kommen.

Die Gemische Aceton-Methylalkohol (Abb. 2c und Tab. 2c) haben einen negativen Volumeneffekt. Hierfür sind immer verhältnismäßig hohe Attraktionskräfte verantwortlich zu machen; Entassoziationsund Packungseffekt $\left(\Delta V_{\mathrm{E}}, \Delta V_{\mathrm{P}}>0\right)$ sind überkompensiert. Es steht zur Diskussion, ob es sich hier um andere Kräfte handelt als solche, die aus einer DipolDipolanziehung resultieren.

Das nach der DCM-Formel berechnete Dipolmoment $\bar{\mu}$ steigt linear mit der Acetonkonzentration an. Beide Substanzen treten also mit einem konstanten Moment auf, und zwar mit dem niedrigsten Wert, der nach der DCM-Formel überhaupt möglich ist und der dem höchsten Assoziationszustand angehört.

Die nach Onsager berechneten $\bar{\mu}$-Werte dagegen, die wieder das mittlere Moment des einzelnen Mole- küls betreffen, haben einen nahezu konstanten Verlauf. Ein schwaches Minimum befindet sich zwischen 0,3 und $0,4 x_{\mathrm{Ac}(\mathrm{M})}$. Nach der Onsagerschen Theorie deutet dieser Kurvenverlauf wieder auf Dipol-Dipolanziehung hin. Angenommen, es liegen in MethanolAceton-Gemischen folgende verschiedene Molekülsorten vor: 1. Methanolmoleküle, die über H-Brücken zu Molekülketten verschiedener Zähligkeit assoziiert sind. (Im unpolaren Lösungsmittel nimmt die Kettenlänge mit der Konzentration ab.) 2. Acetonmoleküle mit nahezu konzentrationsunabhängigem Dipolmoment und Aceton-Einzelmoleküle. Das mittlere Moment des Einzelmoleküls beträgt dabei $\sim 3 \mathrm{D}$. Nun befindet sich das Minimum von $\bar{\mu}$ gerade dort, wo die Alkoholkonzentration noch groß ist und jedenfalls im unpolaren Lösungsmittel auf das Methanolmolekül wegen der H-Brückenbildung noch ein hohes Moment entfällt. Das Aceton hat zwar bei kleinen Konzentrationen ein Moment, das etwas geringer als 3 ist, aber das dürfte hier nicht ausschlaggebend sein. Bei größeren Acetonkonzentrationen jedoch sollte das mittlere Dipolmoment kleiner als die tatsächlichen Werte sein, wenn die Methanolmoleküle die gleichen Eigenschaften beibehalten wie bei Lösung in einer unpolaren Substanz. Andernfalls muß man eine spezifische Wechselwirkung zwischen Aceton und Methanol annehmen, die über die normale Dipol-Dipolanziehung hinausgeht. Es würde sich das in einer Konzentrationsabhängigkeit beider Dipolmomente äußern, von denen in $\bar{\mu}$ ja nur die Resultante beschrieben wird. Verfährt man, entsprechend wie bei Methanol-Chlorbenzol, so, als ob das Moment von Aceton im wesentlichen unverändert bliebe und die spezifische Änderung der zwischenmolekularen Kräfte sich auf das Methanol allein auswirke, so erhält mit $\mu_{\mathrm{Ac}}^{\circ} \sim 3$ das Moment $\mu_{\mathrm{M}(\mathrm{Ac})}$ aus Gl. (13) (s. Abb. 2c).

Dabei zeigt sich nun, daß das Moment von Methanol fast konstant und der Abfall für kleine Konzentrationen verschwunden ist. Das ändert sich auch nicht, wenn man für das Dipolmoment von Aceton die geringe Konzentrationsabhängigkeit bei Lösung in unpolarer Substanz berücksichtigt. Es liegen dann die $\mu_{\mathrm{M}(\mathrm{Ac})}$-Werte etwas über der Kurve $\bar{\mu}_{\text {ons }}$. Demnach verhalten sich die Alkoholmoleküle in Gegenwart von Aceton etwa so, als seien nur arteigene Moleküle vorhanden. Da die Neigung zur Brückenund damit zur Kettenbildung für reines Methanol als bekannt vorausgesetzt werden darf, muß man annehmen, daß die H-Brücken des Methylalkohols 
(OH ... OH-Brücken) durch die Verdünnung mit Aceton zunächst zerstört und dann durch neue, zum Aceton gebildete Brücken $(\mathrm{OH} \ldots \mathrm{O}=\mathrm{C}-$ Brücken $)$ ersetzt werden (s. Abb. 3). Dafür scheint auch der Vergleich der Bindungsenergien zu sprechen; für die $\mathrm{OH} \ldots \mathrm{OH}-$ Brücke beträgt sie $\sim-5000 \mathrm{cal} / \mathrm{Mol}$, für die $\mathrm{OH} \ldots \mathrm{O}=\mathrm{C}$-Brücke jedoch $\sim-7000 \mathrm{cal} / \mathrm{Mol}^{13}$. Es müssen daher die Anziehungskräfte zwischen den artfremden Molekülen größer sein als zwischen den arteigenen. Auf diese Anziehungskräfte ist auch der negative Volumeneffekt zurückzuführen, da ja sowohl Aceton als auch Methanol im Gemisch mit unpolaren Substanzen i. allg. infolge einer Entassoziation positive Volumeneffekte haben.

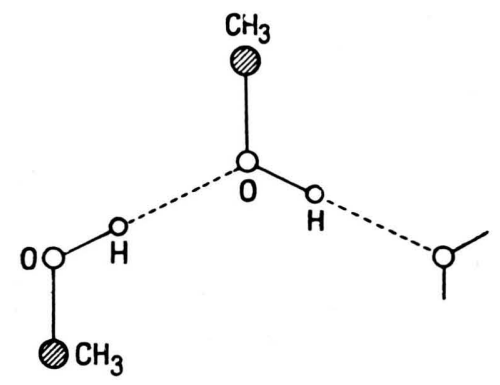

a)

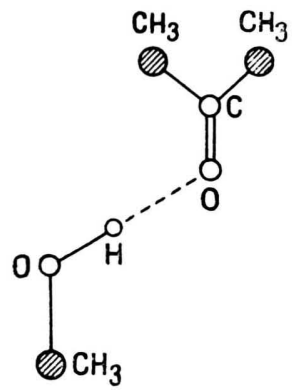

b)
Abb. 3. a) $\mathrm{OH}$... OH-Brückenbindung in Methylalkohol; b) $\mathrm{OH} \ldots \mathrm{O}=\mathrm{C}$-Brückenbindung zwischen Methylalkohol und Aceton.

Stellt man nun ternäre Gemische mit Schwefelkohlenstoff her, indem man zu einer konstanten $\mathrm{Zu}$ sammensetzung Schwefelkohlenstoff-Methanol (welche zweckmäßig außerhalb der Mischungslücke liegt) Aceton hinzufügt, so erhält man für verschiedene Grundmischungen ${ }^{14}$ Schwefelkohlenstoff-Methanol immer das gleiche charakteristische Bild des Volumeneffektes (Abb. 2d und Tab. 2d). Für kleine Acetonkonzentrationen hat der Volumeneffekt ein bei etwa $-0,4 \mathrm{~cm}^{3}$ liegendes Minimum. Von etwa 0,5 Molanteilen Acetonzusatz an verschwindet er fast vollständig. Dieser Verlauf ist deswegen besonders auffällig, weil ohne das Auftreten spezifischer zwischenmolekularer Kräfte die Volumeneffekte additiv sein sollten. Unter dieser Annahme errechnet sich ein Volumeneffekt, der von $x_{\mathrm{Ac}}=0$ bis $x_{\mathrm{Ac}}=1,0$ positiv ist und kontinuierlich abfällt. Der experimentell

13 G. B riegleb u. a., in "Zwischenmolekulare Kräfte“, Verlag G. Braun, Karlsruhe 1949.

14 Untersucht wurde für: $\frac{x_{M}}{x_{M}+x_{C}}=0,83 ; 0,85 ; 0,87$.

\begin{tabular}{|l|c|c|c|c|c|c|}
\hline \multicolumn{1}{|c|}{$x_{\mathrm{Ac}}$} & $x_{\mathrm{M}}$ & $\Delta V_{\mathrm{cm}^{3}}$ & \multicolumn{1}{|c|}{$\varepsilon$} & $\bar{\mu}_{\mathrm{DCM}}$ & $\bar{\mu}_{\text {ons }}$ & $\mu_{\mathrm{M} \text { (Ac)ons }}$ \\
\hline 0 & 0,8551 & $+0,474$ & 28,0 & 1,26 & 2,99 & 2,98 \\
0,8671 & 0,1136 & $-0,009$ & 22,1 & 1,44 & 2,97 & 2,77 \\
0,4329 & 0,4859 & $-0,240$ & 23,1 & 1,35 & 2,87 & 2,80 \\
0,7199 & 0,2395 & $+0,056$ & 22,6 & 1,42 & 2,93 & 2,76 \\
0,2621 & 0,6310 & $-0,311$ & 23,7 & 1,31 & 2,83 & 2,84 \\
0,4961 & 0,4309 & $-0,140$ & 23,0 & 1,37 & 2,89 & 2,79 \\
0,6107 & 0,3329 & 0 & $22,9,3$ & 1,40 & 2,93 & 2,77 \\
0,1681 & 0,7714 & $-0,24$ & 24,75 & 1,29 & 2,86 & 2,88 \\
1,0 & 0 & 0 & 21,7 & 1,47 & 3,00 & - \\
\hline
\end{tabular}

Tab. 2 d. Aceton in Metanol mit $\mathrm{CS}_{2}$ :

$$
\begin{gathered}
\frac{x_{\mathrm{M}}}{x_{\mathrm{M}}+x_{\mathrm{C}}}=0,8551\left(x_{\mathrm{Ac}}+x_{\mathrm{M}}+x_{\mathrm{C}}=1\right) \\
V_{\mathrm{M}}^{20^{\circ}}=40,455 \mathrm{~cm}^{3} ; n_{\mathrm{M}}^{2}=1,818 \\
V_{\mathrm{Ac}}^{20^{\circ}}=73,269 \mathrm{~cm}^{3} ; n_{\mathrm{Ac}}^{2}=2,006 \\
V_{\mathrm{C}}^{20^{\circ}}=60,127 \mathrm{~cm}^{3} ; n_{\mathrm{C}}^{2}=2,68
\end{gathered}
$$

festgestellte Verlauf ist nur so zu verstehen, daß der Zusatz von Aceton wieder eine Brückenbildung $\mathrm{C}=\mathrm{O} \ldots \mathrm{HO}$ mit Methanol zur Folge hat. Das Minimum zeigt durch seine Lage im Konzentrationsbereich eine Stelle an, bei der offenbar gegenüber den anderen Konzentrationen die relativ größte Anzahl von Methanol- und Acetonmolekülen zu Übermolekülen durch $\mathrm{OH} \ldots \mathrm{O}=\mathrm{C}$-Brücken gebunden werden. Für kleinere Acetonkonzentrationen ist der Methanolüberschuß so groß, daß der mit Schwefelkohlenstoff positive Volumeneffekt die wesentliche Rolle spielt; für größere Acetonkonzentrationen macht sich der positive Volumeneffekt bemerkbar, der von den überschüssigen, nicht durch $\mathrm{C}=\mathrm{O} \ldots$ HO-Brücken gebundenen Acetonmolekülen mit Schwefelkohlenstoff herrührt. Gibt man dem Minimum die entsprechende Bedeutung, so wird die relativ größte Anzahl von Aceton-Methanol-Übermolekülen bei etwa 0,2 bis 0,3 Molanteilen Aceton in Methanol gebildet. Diese Zusammensetzung erscheint damit auch als die energetisch günstigste. Dabei steht zur Diskussion, ob die Acetonmoleküle mit den Methylalkoholmolekülen eine $u$. U. räumliche Kette bilden oder ob sie „eingelagert" werden (s. Abb. 4).

Die letztere Möglichkeit erscheint deshalb fraglich, weil doppelte, von doppelt gebundenem Sauerstoff ausgehende Brücken bisher nur als innermolekulare Brücken festgestellt werden konnten ${ }^{15}$. Immerhin tritt die hierfür sprechende „charakteristische“ Konzentration nicht nur bei der Untersuchung des Volumeneffekts, sondern auch bei der Bestimmung

15 s. Bericht v. H o y e r, Z. Elektrochem. angew. physik. Chem. 49, 97 [1943]. 
des relativen Streuvermögens bestimmter RamanLinien von Aceton in Methanol auf ${ }^{16}$.

Das Minimum des Volumeneffekts für die binären Gemische Aceton-Methanol tritt deshalb nicht in gleicher Weise wie für die ternären hervor, da es sich um Úbermoleküle polaren Charakters handelt, zwischen denen es infolge von Dipol-Dipolanziehung auch noch zu kontrahierenden Kräften kommt. Durch den Zusatz des unpolaren Schwefelkohlenstoffs wird die
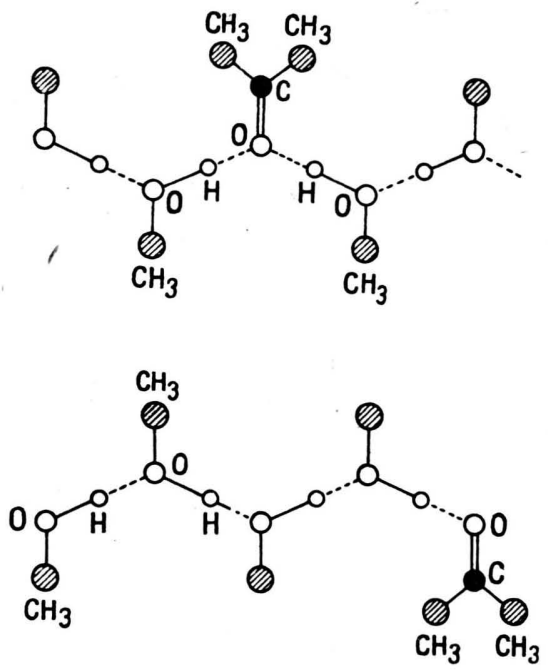

Abb. 4. Möglichkeiten für die $\mathrm{OH} \ldots \mathrm{O}=\mathrm{C}-$ Brückenbindung zwischen Methylalkohol und Aceton.

Wechselwirkung der Übermoleküle untereinander herabgesetzt und die Brückenbildung $\mathrm{OH} \ldots \mathrm{O}=\mathrm{C}$ wird merklicher.

Die Berechnung des auf ein Einzelmolekül im Mittel entfallenden Dipolmoments nach der DCMFormel ergibt wegen des $\mathrm{CS}_{2}$-Zusatzes erwartungsgemäß etwas höher liegende $\bar{\mu}$-Werte als für die binären Gemische Aceton-Methanol. Auch das Ergebnis der nach Onsager berechneten $\bar{\mu}$-Werte unter-

16 M. Joerges u. A. Nikuradse, Z. Naturforschg. 5 a, 25 [1950]. scheidet sich nicht wesentlich von dem für die betreffenden binären Gemische. Nur liegen sowohl die $\bar{\mu}$ als auch die nach $\mathrm{Gl}$. (13) berechneten Werte $\mu_{\mathrm{M}(\mathrm{Ac})}$ etwas niedriger als für Methanol in reinem Aceton, was auf die das Moment $\mu_{\mathrm{M}}$ erniedrigende entassoziierende Wirkung des Schwefelkohlenstoffs zurückzuführen ist. Das große zusätzliche Moment, welches für Methanol auch hier bei kleinen Konzentrationen aus $\operatorname{der} \mathbf{C}=\mathrm{O}$... HO-Brückenbildung resultiert, zeigt sich erwartungsgemäß in der geringen Konzentrationsabhängigkeit von $\mu_{\mathbf{M}(\mathrm{Ac})}$. Eine Auszeichnung der Konzentration 0,2-0,3 Molanteilen Aceton tritt dabei nicht auf. Aber diese Feinheit dürfte auch nach den Voraussetzungen der Onsagerschen Theorie nicht zu erwarten sein.

Mit der Annahme von $\mathrm{C}=\mathrm{O} \ldots \mathrm{OH}-$ Brücken zwischen Aceton- und Methanolmolekülen lassen sich also sowohl der Verlauf der Volumeneffekte als auch die geringe Konzentrationsabhängigkeit der Dipolmomente $\bar{\mu}$ und der Momente $\mu_{\mathrm{M} \text { (Ac) }}$ erklären, wenn man sie nach Onsager berechnet. Das ist aber nur möglich, weil diese Theorie spezielle Assoziationsarten erkennen läßt und die gewöhnliche DipolDipolanziehung extrapoliert. In der DCM-Formel für die Dipolmomente sind alle möglichen Arten von zwischenmolekularen Wechselwirkungen superponiert und daher lassen sich spezielle Assoziationszustände nicht erkennen.

Die Brückenbildung $\mathrm{OH} \ldots \mathrm{O}=\mathrm{C}$ zwischen Methylalkohol und Aceton tritt nun natürlich nicht nur bei der Polarisation bzw. bei Dichtebestimmungen, sondern auch bei der Untersuchung anderer Eigenschaften zutage. So wurde die $\mathrm{C}=\mathrm{O} \ldots \mathrm{OH}$-Brücke bei der Untersuchung der Raman-Linien von AcetonMethanol-Gemischen durch Frequenzerniedrigung der $\mathrm{OH}$-Deformationsfrequenz von Methanol und der $\mathrm{C}=\mathrm{O}$-Doppelbindungsfrequenz von Aceton nachgewiesen ${ }^{16}$.

Wir möchten nicht versäumen, an dieser Stelle Hrn. Prof. Mecke und Hrn. Dr. Reuter für wertvolle Diskussionen zu danken. 